\title{
Variación en la actividad acústica de Hyalinobatrachium fleischmanni (Anura: Centrolenidae) en un ambiente urbano con diferentes niveles de ruido antropogénico
}

\author{
Ana Cecilia Gutiérrez-Vannucchi ${ }^{1}{ }^{*}$, Paulina Rodríguez-Umaña ${ }^{1}$, Luis Sandoval ${ }^{1,2}$ \\ \& Adrián García-Rodríguez $z^{1,3,4}$ \\ 1. Escuela de Biología, Universidad de Costa Rica, CP-11501-2060, San Pedro, San José, Costa Rica; \\ anagv04@gmail.com, rodriguezuma.p@gmail.com \\ 2. Laboratorio de Bioacústica, Escuela de Biología, Universidad de Costa Rica, CP-11501-2060, San Pedro, San José, \\ Costa Rica; biosandoval@hotmail.com \\ 3. Pós-Graduação em Ecologia, Universidade Federal do Rio Grande do Norte, Natal, RN, Brazil, 59078-900; \\ garciar.adrian@gmail.com \\ 4. Departamento de Zoología, Instituto de Biología, Universidad Nacional Autónoma de México, Mexico City, Mexico. \\ * Correspondencia
}

Recibido 20-XI-2017. Corregido 18-I-2019. Aceptado 06-II-2019.

\begin{abstract}
Diel acoustic variation in Hyalinobatrachium fleischmanni (Anura: Centrolenidae) in urban habitat with different anthropogenic noise levels. Acoustic signals produced by animals can be masked by natural or anthropogenic environmental noise. A high proportion of the current distribution of the glass-frog Hyalinobatrachium fleischmanni (Centrolenidae) overlaps with noisy urban habitats, and this may affect the species acoustic communication. In this work, we analyzed the relationship between changes in anthropogenic noise level throughout the night and the diel acoustic variation (vocalization per time) in H. fleischmanni, within three sites with different noise level. We conducted this study in three sites inside the Rodrigo Facio University Campus, San José, Costa Rica in June 2015. We used an automated recorder per site to record glass-frog vocalizations, from 17:00 to 6:00 h, during two consecutive days, every two weeks, for a month. Simultaneously, we measured the noise levels in $\mathrm{dB}$ each hour using a soundmeter. We used linear mixed models to compare the vocalization mean per hour against the noise levels per hour and site. Our results showed that noise levels and H. fleischmanni acoustic activity varied throughout the night. The H. fleischmanni acoustic activity at the noisier site was different from sites with lower noise levels. The highest acoustic activity was found in the less noisy site throughout the sampled period. Although we fail to found a direct relationship between noise level and $H$. fleischmanni acoustic activity, noise level variation affects vocal activity pattern in this species.
\end{abstract}

Key words: acoustic communication; advertisement song; anuran; diel patterns; urban areas.

Gutiérrez-Vannucchi, A. C., Rodríguez-Umaña, P., Sandoval, L. \& García-Rodríguez, A. (2019). Variación en la actividad acústica de Hyalinobatrachium fleischmanni (Anura: Centrolenidae) en un ambiente urbano con diferentes niveles de ruido antropogénico. Revista de Biología Tropical, 67(2) Suplemento, S249-S258.

Para una gran variedad de organismos -desde artrópodos hasta mamíferos- la comunicación acústica es importante en diversos contextos (Bradbury \& Vehrencamp, 2007). Entre las funciones principales de la comunicación acústica están la defensa de territorio, atracción de pareja, indicación de alarma y presencia de alimento (Langmore, 1998; Catchpole \& Slater, 2008; Bradbury \& Vehrencamp, 2011; Herrera-Montes \& Aide, 2011); por lo tanto, transmitir correctamente los mensajes acústicos es fundamental para la comunicación. 
Sin embargo, las señales acústicas pueden ser enmascaradas por el ruido del medio ambiente, generado por ríos, viento, lluvia, otros animales, o fuentes artificiales o antropogénicas (Amézquita et al., 2006; Herrera-Montes \& Aide, 2011; Vargas-Salinas \& Amézquita, 2013), afectando negativamente la comunicación acústica al disminuir la distancia de transmisión (Boncoraglio \& Saino, 2007; Ey \& Fisher, 2009) y degradar la energía y señal (Slabbekoorn \& den Boer-Visser 2006, Warren, Katti, Ermann \& Brazel, 2006). Consecuentemente, varios grupos de animales (ej.: grillos, aves, mamíferos, y anfibios) minimizan el efecto del enmascaramiento mediante cambios en el comportamiento, como incrementar los valores de las frecuencias mínimas e intensidad del canto o algunos de sus elementos (Bermúdez-Cuamatzin, Ríos-Chelén, Gil, \& García, 2011; Lampe, Schmoll, Franzke, \& Reinhold, 2012; Redondo, Barrantes, \& Sandoval, 2013; Hanna, Wilson, Blouin-Demers, \& Mennill, 2014), o cantando a horas con menos ruido (Herrera-Montes, \& Aide, 2011; Roy, Miller, Gottsch, \& Wang, 2011)

En las zonas urbanas, se disminuye el área del hábitat natural para las especies de animales (Harris, 1984; Melles, Glenn, \& Martin, 2003; Biamonte, Sandoval, Chacón, \& Barrantes 2011), y se da un aumento en los niveles de ruido debido a actividades antropogénicas (Slabbekoorn\&Peet,2003; Lengagne, 2008). Ambos aspectos pueden afectar la sobrevivencia, salud, y comunicación de los individuos que persisten en estos ambientes (Barber, Crooks, \& Fristrup, 2010; Hanna et al., 2014). Por lo tanto, comprender cuales especies de animales son más propensos a los efectos del ruido antropogénico y cómo contrarrestan este factor para comunicarse eficientemente, se vuelve importante para proponer planes de manejo, planificación de ciudades, políticas de conservación y entender los cambios en la distribución de las especies (Laiolo, 2010).

Uno de los grupos de animales que se está viendo afectado por el enmascaramiento de sus señales acústicas son los anuros (HerreraMontes \& Aide, 2011), debido a que la mayoría de sus vocalizaciones ocurren por debajo de los $5 \mathrm{kHz}$, rango al cual ocurre también la mayoría del ruido antropogénico (Wood \& Yezerinac, 2006; Hanna et al., 2014). Por lo tanto, es intuitivo pensar que los anuros podrían presentar adaptaciones similares a las que utilizan otros grupos de animales para hacer frente a los efectos de traslape o enmascaramiento acústico cuando viven en ambientes urbanos (VargasSalinas \& Amézquita, 2013; Herrera-Montes \&Aide, 2011; Lampe et al., 2012). Una de las especies de anuros donde se esperaría encontrar diferentes comportamientos para contrarrestar el efecto del ruido antropogénico en la comunicación acústica es Hyalinobatrachium fleischmanni (Centrolenidae), debido a que es una especie común en ríos y arroyos urbanos cercanos a carreteras o zonas de alto tránsito de personas, y cuyo canto se produce entre los 3.8 y los 5.3 kHz (Savage, 2002; Kubicki, 2007), traslapando con el ruido antropogénico, que se produce por debajo de los $5 \mathrm{kHz}$ (Wood \& Yezerinac, 2006; Hanna et al., 2014). Debido a que el ruido antropogénico generado en estas áreas puede llegar a enmascarar importantes señales sociales dentro de las poblaciones urbanas de estas especie (Herrera-Montes \& Aide, 2011), consideramos que es una especie adecuada para estudiar el efecto del ruido en la comunicación de anuros.

Nuestro objetivo con este estudio es analizar si existe una relación entre la variación de los niveles de ruido antropogénico a través de la noche y la actividad vocal (cantidad de vocalizaciones por unidad de tiempo) de $H$. fleischmanni, a lo largo de un gradiente de ruido urbano. Si el ruido influye en la actividad vocal de $H$. fleischmanni predecimos que a mayor nivel de ruido antropogénico menor la actividad vocal de esta especie en la noche. También predecimos que a las horas con menos ruido es donde habrá una mayor actividad vocal, ya que esto facilitaría la comunicación acústica.

\section{MATERIALES Y MÉTODOS}

Sitio de estudio: Realizamos las grabaciones durante el mes de junio de 2015, dentro 
del campus universitario Rodrigo Facio, San Pedro, San José $\left(09^{\circ} 56^{\prime}\right.$ N \& 84 $4^{\circ} 05^{\prime}$ W, 1200 m.s.n.m.) (Fig. 1). El sitio de estudio es una mezcla de zonas verdes, edificios, carreteras internas y una reserva ecológica de bosque secundario, todos rodeados por una de las principales carreteras del país al oeste (circunvalación este) y carreteras de menor tráfico al norte, sur y este. Lo anterior produce que los niveles de ruido varíen dentro del área de estudio y a lo largo de la quebrada Los Negritos, que atraviesa todo el campus, dividiéndolo en áreas similares. Esta quebrada tiene aproximadamente 5 $\mathrm{m}$ de ancho y $800 \mathrm{~m}$ de largo dentro del área de estudio. Además presenta un cauce intermitente a lo largo del año, secándose completamente en la época seca y con un cauce constante durante la época lluviosa, cuando se realizó el estudio.

Realizamos las grabaciones en tres puntos a lo largo de la quebrada Los Negritos (Fig. 1). Sitio Económicas, es el que aparentemente presenta mayores niveles de ruido por estar más cercano a la carretera de mayor tránsito y la entrada principal del campus universitario. Además, posee una alta abundancia de árboles con diámetros a la altura del pecho mayores a $10 \mathrm{~cm}$ como Ficus sp. (Moraceae), Cedrella odorata (Meliaceae), Erythrina poeppigiana (Fabaceae), Eucalyptus sp. (Myrtaceae) o bambúes gigantes (Poaceae) mezclada con enredaderas, arbustos y plantas ornamentales como zingiberaces, costaces y bambues pequeños. Sitio Bosquecito, es el que aparentemente presenta menor nivel de ruido al encontrarse en el interior de la reserva ecológica y al centro del campus, y cuenta con acceso restringido de personas. La vegetación de árboles en términos de especies y abundancia de este sitio fue muy similar a la del sitio Económicas, pero con mayor diversidad de arbustos. Sitio Música, es el que aparentemente presenta niveles de ruido intermedio por ubicarse al extremo norestes del campus, y poseer menor número de autos transitando cerca. La vegetación es abierta al poseer muy pocos árboles en el borde del cauce de la quebrada y no tener arbustos por ser un área de jardines.

Grabación de sonidos y medición de ruido: Colocamos en cada sitio una grabadora

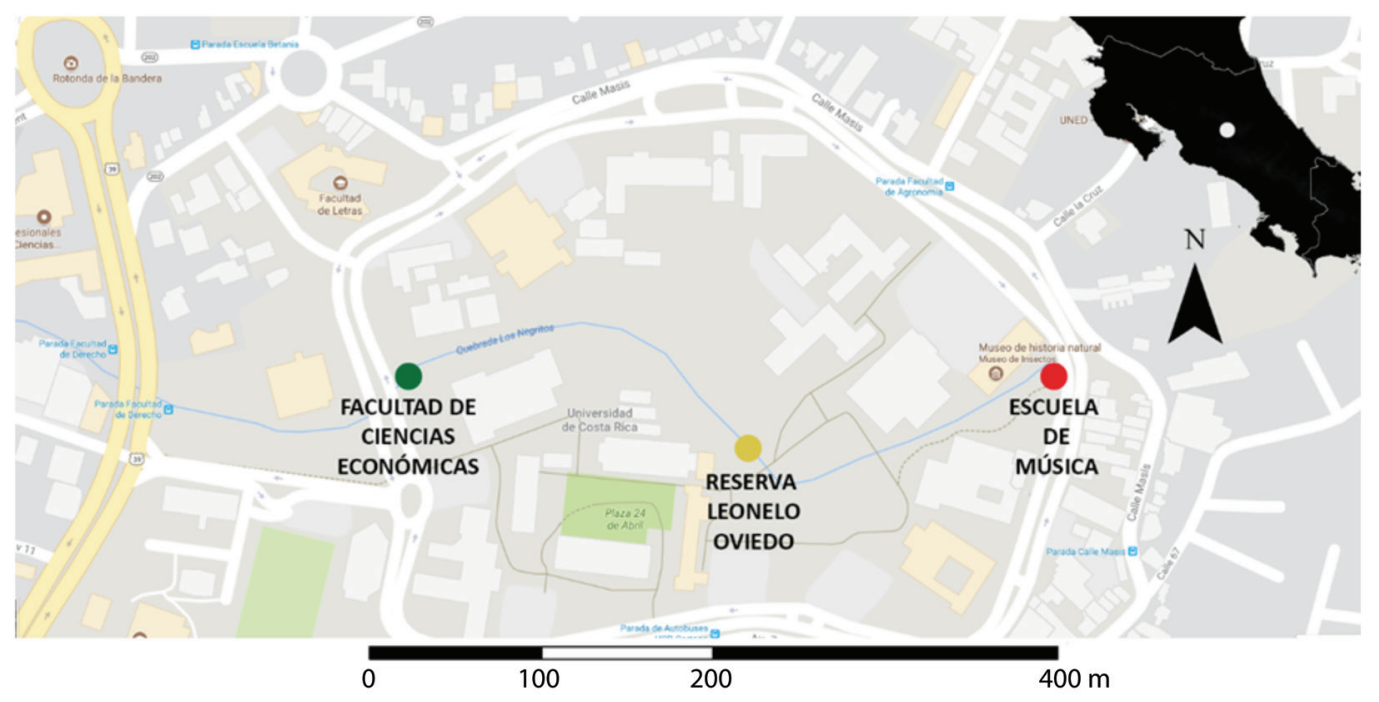

Fig. 1. Mapa del campus Universitario Rodrigo Facio con los tres sitios utilizados para grabar la actividad vocal de la rana de vidrio Hyalinobatrachium fleischmanni y medir los niveles de ruido. En la esquina superior derecha se observa el mapa de Costa Rica.

Fig. 1. Rodrigo Facio University Campus map with the three sites used for recording of Hyalinobatrachium fleischmanni glass-frog (Centrolenidae) vocal activity and measured of noise levels. Costa Rica's map on the upper right corner. 
Song Meter SM2+ (WildlifeAcoustics), con dos micrófonos omnidireccionales SMX-II (WildlifeAcoustics) y configuradas a una tasa de muestreo de $32 \mathrm{kHz}$ y una precisión de 16 bits en un formato WAV. Cada grabación la realizamos en estéreo. Las grabadoras fueron colocadas en árboles al borde de la quebrada entre 0.5 y $1 \mathrm{~m}$ de altura, y cerca de posibles perchas para las ranas (arbustos con hojas colgantes sobre el cauce de la quebrada). Los tres sitios para colocar las grabadoras fueron seleccionados porque los niveles de ruido a los que están expuestos aparentemente parecen variar. Las grabadoras fueron configuradas para grabar de las 17:00 a las 06:00, periodo durante el que grabaron $59 \mathrm{~min}$ por hora. En cada sitio grabamos dos días consecutivos, cada dos semanas, por un mes, para un total de cuatro días por sitio, equivalentes a 52 horas en cada punto de grabación. Además, las grabadoras fueron colocadas lo mismos cuatro días en los tres sitios.

Los datos de ruido los obtuvimos durante dos días, siempre el día previo a realizar las grabaciones. Para tal fin utilizamos ocho puntos alrededor de cada sitio de grabación de los cuales cuatro se encontraron a $10 \mathrm{~m}$ y cuatro a $20 \mathrm{~m}$ de la grabadora y al menos a $10 \mathrm{~m}$ entre sí. En cada uno de estos puntos medimos los valores de ruido mínimo y máximo cada hora de las 17:00 a 06:00. Para la toma de la medición de ruido empleamos un Sonómetro SperScientific 840014 mini soundmeter (ámbito de medidas $32-130 \mathrm{~dB}$, en respuesta rápida y con medición tipo A). Posteriormente estimamos el promedio del valor de ruido para cada hora por punto.

Actividad vocal: Utilizamos el programa Raven 1.4 (Cornell Lab of Ornithology, Ithaca, Nueva York, EEUU), para anotar la cantidad de vocalizaciones que fueron emitidas por hora de 17:00 a las 06:00 en cada punto de grabación. Aunque estas ranas únicamente vocalizan durante la noche, algunas veces si llueve muy fuerte durante la tarde pueden empezar a vocalizar después del aguacero (Savage, 2002; Kubicki, 2007). Sin embargo, durante nuestro periodo de muestreo no llovió durante la tarde por lo tanto, estamos seguros que grabamos todo el periodo de actividad de los individuos en cada sitio. Debido a que no fue posible discernir la cantidad de individuos que estaban vocalizando a partir de los sonogramas (representación visual de los sonidos), es intuitivo pensar que existe relación positiva entre la cantidad de individuos y la cantidad de vocalizaciones contadas en cada hora de grabación por punto. No fue posible discernir la cantidad de machos debido a que pudieron haber varios individuos a distancias similares de la grabadora horizontal y verticalmente, lo que produciría observar cantos con la misma cantidad de energía en los sonogramas y por lo tanto, asignárselos erróneamente a un único macho (subestimando la cantidad de individuos). Además, los machos tienden a moverse y cambiar su posición durante el canto (obs. pers.), por lo que este cambio en la dirección va a causar una diferencia de energía en la señal captada por el micrófono, y podría confundirse con señales de machos distintos (sobreestimando la cantidad de individuos). Por lo tanto nuestra medida de actividad y comparación entre sitios es la cantidad de vocalizaciones promedio por hora por sitio.

Análisis estadístico: Hicimos un análisis linear mixto para determinar si hay variación en el valor del ruido por hora por sitio. En este modelo utilizamos como variable dependiente el valor de ruido por hora, como variables independientes la hora del día, el sitio y la interacción entre ambas, y como variable aleatoria la fecha del muestreo. Realizamos otro análisis linear mixto para determinar si hay variación en la cantidad de vocalizaciones por hora por sitio. En este modelo utilizamos como variable dependiente el número de vocalizaciones por hora, como variables independientes la hora del día, el sitio y la interacción entre ambas, y como variable aleatoria la fecha del muestreo. Además, realizamos una correlación entre la cantidad promedio de vocalizaciones por hora y el ruido promedio por hora. Los análisis los realizamos utilizando el programa JMP versión 
7.0 (SAS Institute, Cary, NC, EEUU) para realizar nuestros análisis estadísticos.

\section{RESULTADOS}

Encontramos que el sitio con mayor nivel de ruido promedio fue Económicas $\left(\mathrm{F}_{2,509}=\right.$ 5.64, $\mathrm{P}=0.004)$, mientras que los sitios de Música y Bosquecito presentaron un nivel de ruido similar (Fig. 2). El nivel de ruido promedio varió a lo largo de las horas de muestreo $\left(\mathrm{F}_{13,508}=16.21, \mathrm{P}<0.001\right)$, presentando los valores máximos de ruido de las 17:00 a las 21:00 y de nuevo a las 06:00. Los valores mínimos de ruido se registraron de 01:00 a 04:00 (Fig. 2). Además, encontramos que las horas a las que ocurren los valores mayores de ruido promedio variaron entre sitios $\left(\mathrm{F}_{26,507}\right.$ $=4.94, \mathrm{P}<0.001)$. En el sitio Económicas, el mayor nivel de ruido lo medimos entre las
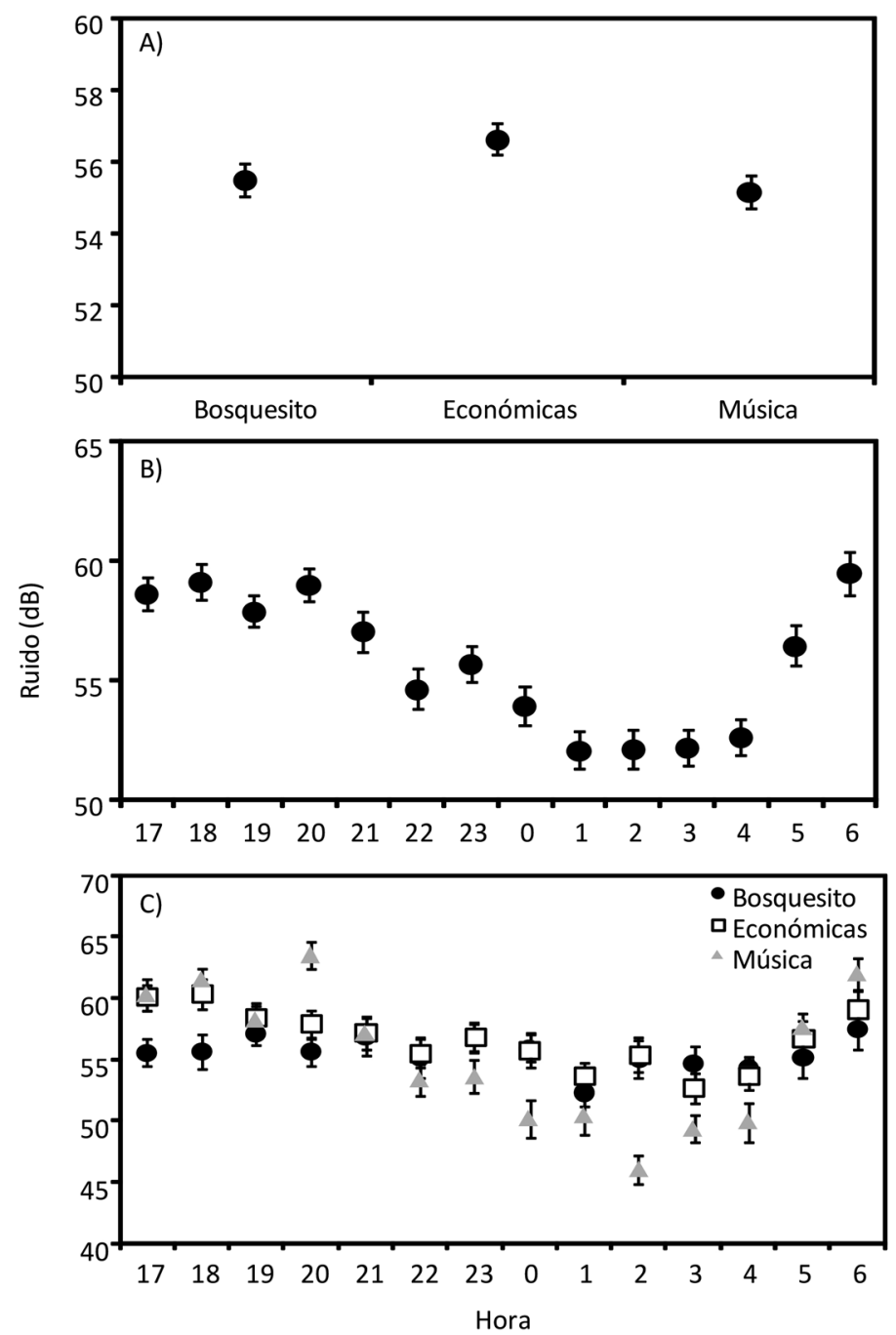

Fig. 2. Niveles de ruido promedio ( \pm error estándar) en tres sitios del Campus Universitario Rodrigo Facio (A), a lo largo de la noche (B), y en la interacción entre sitio y hora (C).

Fig. 2. Average noise levels ( \pm standar error) at three sites of Rodrigo Facio University Campus (A), throughout night (B), and the interaction between sites and hour $(\mathrm{C})$. 
17:00 y las 18:00 y el menor nivel de ruido se dio a las 03:00 (Fig. 2). En el sitio Música, el mayor nivel de ruido lo medimos a las 20:00, el menor nivel de ruido a las 02:00 (Fig. 2). En el sitio Bosquecito, el mayor nivel de ruido lo medimos a las 19:00 y 06:00 y el menor nivel de ruido fue la 01:00 (Fig. 2).

Encontramos que la mayor actividad vocal fue en el Bosquecito $\left(\mathrm{F}_{2,68}=5.38, \mathrm{P}=0.007\right)$, mientras que Música y Económicas presentaron un nivel de actividad vocal menor y similar entre ellos (Fig. 3), aunque variaron en la cantidad de vegetación. El nivel de actividad vocal general también varió a lo largo del periodo de muestreo $\left(\mathrm{F}_{13,66}=1.94, \mathrm{P}=0.04\right)$, presentando los valores mayores de actividad vocal entre las 20:00 y 21:00 h y el menor a las 17:00 h (Fig. 3). Finalmente, encontramos que no hubo interacción entre las horas y la actividad vocal por sitio $\left(\mathrm{F}_{26,66}=0.94, \mathrm{P}=0.56\right.$; Fig. 3$)$, aunque la mayor cantidad de vocalizaciones promedio para el Bosquecito y Música fue a las 21:00
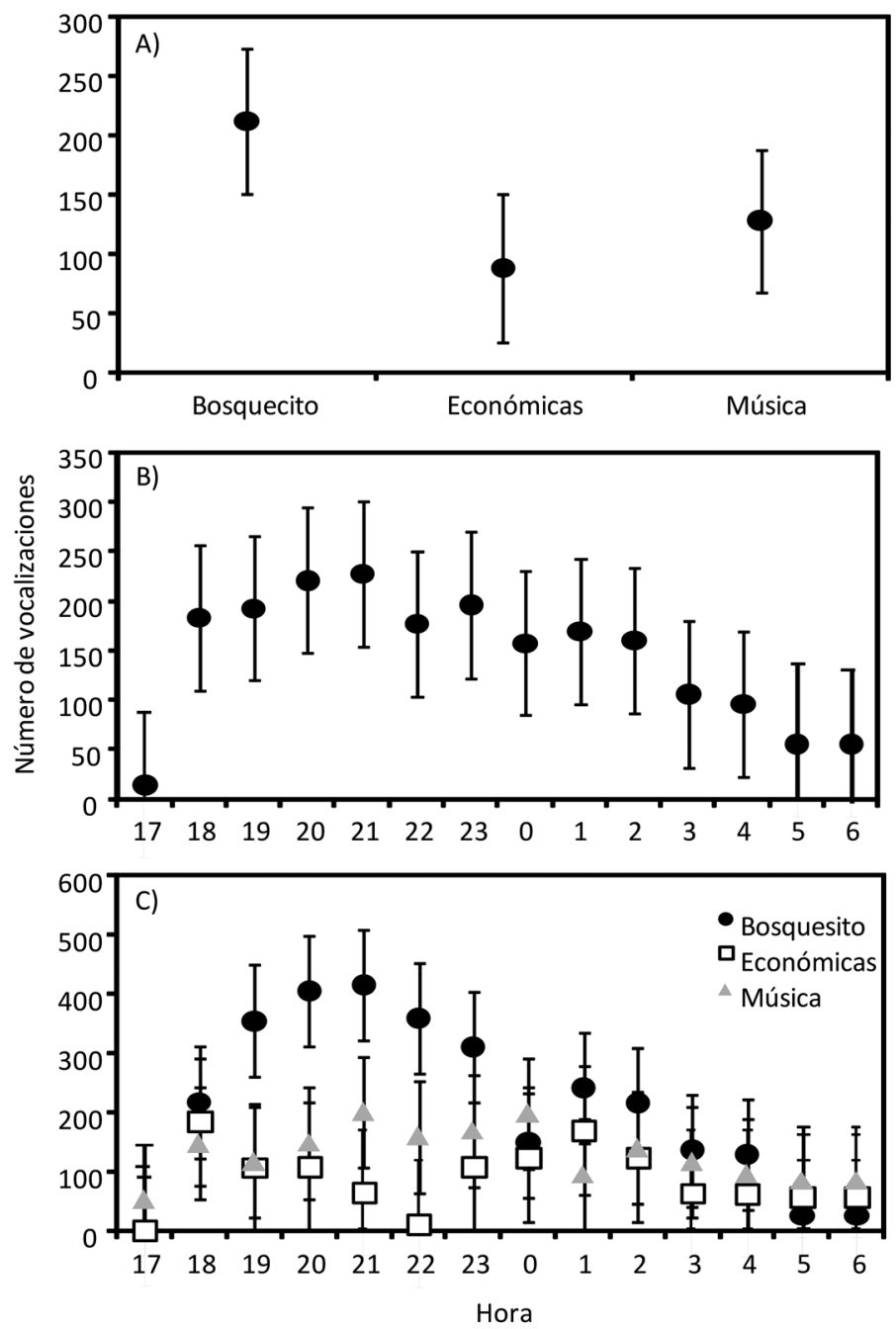

Fig. 3. Número de vocalizaciones promedio ( \pm error estándar) de machos de Hyalinobatrachium fleischmanni en tres sitios del Campus de la Universidad de Costa Rica (A), a lo largo de la noche (B), y en la interacción entre sitio y hora (C).

Fig. 3. Average vocal activity ( \pm standar error) of Hyalinobatrachium fleischmanni males at three sites of Rodrigo Facio University Campus (A), throughout night (B), and the interaction between sites and hour (C). 
y para Económicas a las 18:00 (Fig. 3). No encontramos una correlación entre la cantidad de ruido en cada sitio por hora y la actividad vocal de las ranas $(\mathrm{r}<|0.18|, \mathrm{P}>0.52$ para todas las comparaciones).

\section{DISCUSIÓN}

Nuestros resultados demuestran que, tanto el ruido como la actividad vocal de $H$. fleischmanni variaron a lo largo de la noche, aunque, no hubo una relación entre la cantidad de ruido y la actividad vocal de la especie en ninguno de los tres sitios. Esto contrasta con nuestras predicciones y lo reportado en la literatura, ya que aunque el ruido antropogénico tiene el potencial de enmascarar señales acústicas (Barber et al., 2010; Halfwerk et al., 2011) como las de H. fleischmanni (que cantan por debajo de los $5 \mathrm{kHz}$ ), no encontramos dicho efecto en este estudio. No obstante la mayor actividad vocal a lo largo de toda la noche la registramos en el sitio Bosquecito, que fue el que presentó menor nivel de ruido.

Aunque estudios previos sugieren que el ruido del tráfico puede inhibir la producción de vocalizaciones en anuros llevando a un patrón de espaciamiento temporal, donde los individuos vocalizan en momentos en que el ruido ambiental es menor (Sun \& Narins, 2005; Vargas-Salinas \& Amézquita, 2013), en nuestro caso no hubo tal relación. Esto se puede deber a que la contaminación acústica enmascara especialmente vocalizaciones que se emiten con frecuencias por debajo de los $5 \mathrm{kHz}$ (Gerhardt \& Klump, 1988; Brumm \& Slabbekoorn, 2005; Vargas-Salinas \& Amézquita, 2013). Pero en el caso de $H$. fleischmanni las vocalizaciones de los machos presentan una frecuencia mínima que varía entre 3.8 y $4.5 \mathrm{kHz}$ (Savage, 2002), cercana al límite superior de las frecuencias del ruido antropogénico (Wood \& Yezerinac, 2006; Hanna et al., 2014). Este poco traslape entre frecuencias podría justificar que no haya una relación entre los valores de la actividad vocal y los del ruido en el sitio de estudio, por lo tanto el ruido no estaría interfiriendo en la comunicación acústica de esta especie. Una situación similar se presenta en la rana Pseudacris crucifer donde tampoco se encontró una relación entre el aumento de la tasa de llamadas y el aumento en la cantidad de ruido (Hanna et al., 2014). Variar la tasa de canto es una estrategia aparentemente común en otros anuros, pues aumenta la probabilidad de detectabilidad por parte de los receptores (Penna, Pottstock, \& Velásquez, 2005; Kaiser \& Hammers, 2009).

La diferencia en los picos de actividad vocal entre sitios, se puede deber a dos causas principales, la cantidad de vegetación y el nivel de ruido en cada sitio. Por ejemplo, los sitios Bosquecito y Música, tuvieron un nivel de ruido bajo y similar entre ellos, variaron en la cantidad de vegetación (mayor en Bosquecito), y presentaron patrones de actividad vocal similar (picos y valles a horas similares, Fig. 2 ), pero con cantidades mayores de vocalizaciones por hora en sitio Bosquecito. La mayor cobertura vegetal de sitio Bosquecito en comparación con sitio Música, puede estar creando mejores variables microclimáticas como mayor humedad, temperatura más constante, y menor grado de iluminación, características que se ha visto que influyen en el aumento de la actividad acústica de otros anuros (Alonso, Rodríguez-Gómez, \& Estrada,2001; Oseen \& Wassersug, 2002; Ospina, Villanueva-Rivera, Corrada-Bravo, \& Aide, 2013). Adicionalmente, niveles mayores de humedad como los que puede haber en el sitio Bosquecito en comparación con el sitio Música favorecen el aumento en la cantidad de cantos que producen los machos de H. fleischmanni, ya que si la humedad relativa baja del $93 \%$ la actividad vocal también decrece drásticamente (Hayes, 1991). Una mayor densidad de vegetación puede aumentar la cantidad de perchas adecuadas (hojas colgantes sobre el aguaentre 1 y 5 m; Savage, 2002; Kubicki, 2007) para cantar por parte de los machos y para ovopositar por parte de las hembras. Por lo que el número de machos en este sitio podría ser mayor y por lo tanto la actividad vocal también, si existe una relación positiva entre ambos.

Sin embargo, sitio Bosquecito y Económicas fueron similares en la abundancia de 
vegetación, pero variaron en la cantidad y los patrones de actividad, así como en los niveles de ruido (Figs. 2 y 3). Por lo tanto, a pesar de que no se encontró una relación directa entre los niveles de ruido ambiental con la actividad vocal de $H$. fleischmanni, si observamos que los patrones de actividad variaron cuando varió el ruido. Esto se encontró para el sitio Económicas, que es el que presentó mayor ruido y el único con un patrón de actividad acústica distinto, vocalizando mayormente en las horas de más ruido (18:00). La causa de esta diferencia entre sitios es desconocida, pero se podría deber a que retrasar la actividad vocal para cantar en los momentos con menos ruido (más tarde en la noche) puede disminuir la probabilidad de que lleguen hembras a copular, especialmente si existen otros machos en las cercanías vocalizando desde el inicio de la noche a los cuales las hembras detectarían primero. De este modo, es posible que los machos de $H$. fleischmanni en sitio Económicas, que es el más ruidoso, estén modificando las características espectro-temporales de sus vocalizaciones (ej.: incrementando la frecuencia mínima, la cantidad de energía, o duración). Esto ocurre en otros anuros que habitan sitios ruidosos (Penna et al., 2005; Lengagne, 2008; Kaiser \& Hammers, 2009; Cunnington \& Fahrig, 2010; Gross, Pasinelli, \& Kunc, 2010; Both \& Grant, 2012; Vargas-Salinas \& Amézquita, 2013; Hanna et al., 2014), los cuales incrementan sus frecuencias en lugar de disminuir la actividad vocal o cambiar los patrones de actividad para facilitar su comunicación. Sin embargo nosotros no medimos esas características y sería un trabajo a realizar en el futuro para comprender este patrón observado.

En conclusión, a pesar de que no se encontró una relación directa entre el nivel de ruido antropogénico y la actividad acústica de $H$. fleischmanni, sí observamos un efecto de este en los patrones de actividad de la especie. Las variables ambientales parecen tener un papel importante en la actividad acústica de estas ranas, especialmente la cobertura boscosa y posiblemente la humedad aunque esta última no fue medida. Consideramos que deben tomarse en cuenta más variables tanto bióticas como abióticas en estudios futuros, por ejemplo la fase lunar, características de la quebrada y cantidad de iluminación, entre otras; ya que sólo el ruido no explica completamente los patrones de llamadas encontrados en este trabajo. Lo anterior sugiere que los factores ambientales y ruido antropogénico, podrían estar definiendo de manera conjunta los patrones de actividad acústica para esta especie. La información anterior resulta importante ya que brindan información para el manejo de remanentes de zonas naturales o de importancia para la reproducción de distintas especies de anfibios en las zonas urbanas, así como para la toma de decisiones sobre control o manejo de los niveles de ruido que podrían estar afectando la reproducción, y por ende sobrevivencia, de distintas especies similares a H. fleischmanni.

Declaración de ética: los autores declaran que todos están de acuerdo con esta publicación y que han hecho aportes que justifican su autoría; que no hay conflicto de interés de ningún tipo; y que han cumplido con todos los requisitos y procedimientos éticos y legales pertinentes. Todas las fuentes de financiamiento se detallan plena y claramente en la sección de agradecimientos. El respectivo documento legal firmado se encuentra en los archivos de la revista.

\section{AGRADECIMIENTOS}

A los estudiantes del curso de Bioacústica de la Escuela de Biología de la Universidad de Costa Rica 2015, que colaboraron en tomar los datos de ruido por hora en los sitios de estudio. A la Oficina de Seguridad y Tránsito de la Universidad por facilitar los muestreos nocturnos. También agradecemos al Laboratorio de Bioacústica de la Escuela de Biología por facilitarnos el equipo utilizado para este trabajo. LS agradece a Vicerrectoría de Investigación por el apoyo al proyecto número N-111-B5-241 y a la Escuela de Biología por el medio tiempo de nombramiento. AGR es actualmente financiado por una beca Postdoctoral de la Dirección 
General de Asuntos del Personal Académico (DGAPA) en el Instituto de Biología de la Universidad Nacional Autónoma de México.

\section{RESUMEN}

Las señales acústicas producidas por animales pueden ser enmascaradas por el ruido del medio ambiente en que se encuentran, tanto de origen natural como antropogénico. Una especie que puede ver afectada su comunicación por el ruido medio ambiental es la rana de vidrio Hyalinobatrachium fleischmanni (Centrolenidae), porque la distribución actual de esta especie en gran parte se encuentra en ambientes urbanos con altos niveles de ruido. En este trabajo analizamos si existe una relación entre la variación de los niveles de ruido antropogénico a través de la noche y la actividad vocal (cantidad de vocalizaciones por unidad de tiempo) de H. fleischmanni, a lo largo de un gradiente de ruido urbano. El trabajo lo realizamos en tres sitios dentro del Campus Universitario Rodrigo Facio, San José, Costa Rica durante el mes de junio de 2015. Para contabilizar la actividad vocal de la especie en cada sitio instalamos grabadores programables de 17:00 a las 6:00 por dos días consecutivos cada dos semanas. De forma simultánea, cuantificamos el ruido en $\mathrm{dB}$ cada hora a lo largo del periodo de grabación utilizando sonómetros. Comparamos la abundancia promedio de vocalizaciones por hora contra los niveles de ruido promedio por hora $\mathrm{y}$ sitio utilizando modelos lineales mixtos. Nuestros resultados demuestran que, tanto el ruido como la actividad vocal de H. fleischmanni variaron a lo largo de la noche. Sin embargo, encontramos que en el sitio más ruidoso el patrón de actividad de H. fleischmanni difiere de los dos sitios menos ruidosos.El sitio menos ruidoso fue el que presento una mayor actividad vocal a lo largo de todo el periodo de muestreo. A pesar de que no se encontró una relación directa entre el nivel de ruido antropogénico y la actividad acústica de $H$. fleischmanni, sí observamos un efecto de los niveles de ruido en los patrones de actividad de la especie.

Palabras claves: anuros; área urbana; canto de anuncio; ciclos de actividad; comunicación acústica.

\section{REFERENCIAS}

Alonso, R., Rodríguez-Gómez, A., \& Estrada, A. R. (2001). Patrones de actividad acústica y trófica de machos cantores de Eleutherodactylus eileenae (Anoura: Leptodactylidae). Revista Española de Herpetología, $15,45-52$.

Amézquita, A., Hödl, W., Lima, A. P., Castellanos, L., Erdtmann, L., \&Araújo, M. C. (2006). Masking interference and the evolution of the acoustic communication system in the Amazonian dendrobatid frog Allobates femoralis. Evolution, 60, 1874-1887.
Barber, J. R., Crooks, K. R., \& Fristrup, K. M. (2010). The costs of chronic noise exposure for terrestrial organisms. Trends in Ecology \& Evolution, 25, 180-189.

Bermúdez-Cuamatzin, E., Ríos-Chelén, A. A., Gil, D., \& García, C. M. (2011). Experimental evidence for realtime song frequency shift in response to urban noise in a passerine bird. Biology Letters, 7, 36-38.

Biamonte, E., Sandoval, L., Chacón, E., \& Barrantes, G. (2011). Effect of urbanization on the avifauna in a tropical metropolitan area. Landscape Ecology, 26, 183-194.

Boncoraglio, G., \& Saino, N. (2007). Habitat structure and the evolution of bird song: a meta-analysis of the evidence for the acoustic adaptation hypothesis. Functional Ecology, 21, 134-142.

Both, C., \& Grant, T. (2012). Biological invasions and the acoustic niche: the effect of bullfrog calls on the acoustic signals of white-banded tree frogs. Biology Letters, 8, 714-716.

Bradbury, J. W., \& Vehrencamp, S. L. (2011). Principles of animal communication. Sunderland: Sinauer.

Brumm, H., \& Slabbekoorn, H. (2005). Acoustic communication in noise. Advances in the Study of Behavior, 35, 151-209.

Catchpole, C. K., \& Slater, P. J. (2003). Bird song: biological themes and variations. Cambridge: Cambridge University Press.

Cunnington, G. M., \& Fahrig, L. (2010). Plasticity in the vocalizations of anurans in response to traffic noise. Acta Oecologica, 36, 463-470.

Ey, E., \& Fischer, J. (2009). The "acoustic adaptation hypothesis" -a review of the evidence from birds, anurans and mammals. Bioacoustics, 19, 21-48.

Gerhardt, H. C., \& Klump, G. M. (1988). Masking of acoustic signals by the chorus background noise in the green tree frog: a limitation on mate choice. Animal Behaviour, 36, 1247-1249.

Gross, K., Pasinelli, G.,\&Kunc, H. P. (2010). Behavioral plasticity allows short-term adjustment to a novel environment. American Naturalist, 176, 456-464.

Halfwerk, W., Bot, S., Buikx, J., van der Velde, M., Komdeur, J., ten Cate, C., \& Slabbekoorn, H. (2011). Lowfrequency songs lose their potency in noisy urban conditions. Proceedings of the National Academy of Sciences, 35, 14549-14554.

Hanna, D. E., Wilson, D. R., Blouin-Demers, G., \& Mennill, D. J. (2014). Spring peepers Pseudacris crucifer modify their call structure in response to noise. Current Zoology, 60, 438-448. 
Harris, L. D. (1984). The fragmented forest: island biogeography theory and the preservation of biotic diversity. Chicago: University of Chicago Press.

Hayes, M. P. (1991). A study of clutch attendance in the Neotropical frog Centrolenella fleischmanni (Anura: Centrolenidae) (Doctoral dissertation). University of Miami, USA.

Herrera-Montes, M. I., \& Aide, T. M. (2011). Impacts of traffic noise on anuran and bird communities. Urban Ecosystem, 14, 415-427.

Kaiser, K., \& Hammers, J. L. (2009). The effect of anthropogenic noise on male advertisement call rate in the neotropical treefrog, Dendropsophus triangulum. Behaviour, 146, 1053-1069.

Kubicki, B. (2007). Ranas de vidrio Costa Rica. Heredia, Costa Rica: INBio.

Laiolo, P. (2010). The emerging significance of bioacoustics in animal species conservation. Biological Conservation, 143, 1635-1645.

Lampe, U., Schmoll, T., Franzke, A., \& Reinhold, K (2012). Staying tuned: grasshoppers from noisy roadside habitats produce courtship signals with elevated frequency components. Functional Ecology, $26,1348-1354$

Langmore, N. E. (1998). Functions of duet and solo songs of female birds. Trends in Ecology \& Evolution, 13, 136-140.

Lengagne, T. (2008). Traffic noise affects communication behaviour in a breeding anuran, Hyla arborea. Biological Conservation, 141, 2023-2031.

Melles, S., Glenn, S., \& Martin, K. (2003). Urban bird diversity and landscape complexity: species-environment associations along a multiscale habitat gradient. Conservation Ecology, 7, 5.

Oseen, K. L., \& Wassersug, R. J. (2002). Environmental factors influencing calling in sympatric anurans. Oecologia, 133, 616-625.
Ospina, O. E., Villanueva-Rivera, L. J., Corrada-Bravo, C. J., \& Aide, T. M. (2013). Variable response of anuran calling activity to daily precipitation and temperature: implications for climate change. Ecosphere, 4, 1-12.

Penna, M., Pottstock, H., \& Velásquez, N. (2005). Effect of natural and synthetic noise on evoked vocal responses in a frog of the temperate austral forest. Animal Behaviour, 70, 639-651.

Redondo, P., Barrantes, G., \& Sandoval, L. (2013). Urban noise influences vocalization structure in the House Wren Troglodytes aedon. Ibis, 155, 621-625.

Roy, S., Miller, C. T., Gottsch, D., \& Wang, X. (2011). Vocal control by the common marmoset in the presence of interfering noise. Journal of Experimental Biology, 214, 3619-3629.

Savage, J. M. (2002). The Amphibians and Reptiles of Costa Rica. Chicago: University of Chicago Press.

Slabbekoorn, H., \& den Boer-Visser, A. (2006). Cities change the songs of birds. Current biology, 16, 2326-2331.

Slabbekoorn, H., \& Peet, M. (2003). Bird's sing at a higher pitch in urban noise: Great tits hit the high notes to ensure that their mating calls are heard above the city's din. Nature, 424, 267-267.

Sun, J. W. C., \& Narins, P. M. (2005). Anthropogenic sounds differentially affect amphibian call rate. Biological Conservation, 121, 419-427.

Vargas-Salinas, F., \& Amézquita, A. (2013). Traffic noise correlates with calling time but not spatial distribution in the threatened poison frog Andinobates bombetes. Behaviour, 150, 569-584.

Warren, P. S., Katti, M., Ermann, M., \& Brazel, A. (2006). Urban bioacoustics: it's not just noise. Animal behaviour, 71, 491-502.

Wood, W. E., \& Yezerinac, S. M. (2006). Song sparrow (Melospiza melodia) song varies with urban noise. Auk, 123, 650-659. 